\title{
Clinical utility gene card for: hypertrophic cardiomyopathy (type 1-14)
}

\author{
Yigal M Pinto ${ }^{\star, 1}$, Arthur AAM Wilde ${ }^{1}$, Ingrid AW van Rijsingen ${ }^{1}$, Imke Christiaans ${ }^{2}$, \\ Ronald H Lekanne Deprez ${ }^{2}$ and Perry M Elliott ${ }^{3}$
}

European Journal of Human Genetics (2011) 19, doi:10.1038/ejhg.2010.243; published online 26 January 2011

\section{DISEASE CHARACTERISTICS}

1.1 Name of the disease (synonyms)

Hypertrophic cardiomyopathy (HCM CMH), familial HCM CMH, ventricular hypertrophy, hereditary asymmetric septal hypertrophy, hypertrophic obstructive cardiomyopathy (HOCM), and hypertrophic subaortic stenosis.

\subsection{OMIM\# of the disease}

192600 Cardiomyopathy, familial hypertrophic; $\mathrm{CMH}$.

115195 Cardiomyopathy, familial hypertrophic, 2; CMH2.

115196 Cardiomyopathy, familial hypertrophic, 3; CMH3.

115197 Cardiomyopathy, familial hypertrophic, 4; CMH4. 600858 Cardiomyopathy, familial hypertrophic, 6; CMH6.

+191044 Cardiomyopathy, familial hypertrophic, 7; CMH7. 608751 Cardiomyopathy, familial hypertrophic, 8; CMH8. ${ }^{+} 191044$ Cardiomyopathy, familial hypertrophic, 9; CMH9. 608758 Cardiomyopathy, familial hypertrophic, 10; CMH10. 612098 Cardiomyopathy, familial hypertrophic, 11; CMH11. 612124 Cardiomyopathy, familial hypertrophic, 12; CMH12. 613243 Cardiomyopathy, familial hypertrophic, 13; CMH13. 613251 Cardiomyopathy, familial hypertrophic, 14; CMH14. 613243 Cardiomyopathy, familial hypertrophic, 15; CMH15.

1.3 Name of the analysed genes or DNA/chromosome segments 1. Sarcomeric protein genes:

a. Cardiac myosin-binding protein-C gene (MYBPC-3) locus 11p11.2.

b. Cardiac beta-myosin heavy chain gene (MYH7) locus 14q11.2-q12.

c. Troponin T gene (TNNT2) locus 1q32.

d. Troponin I gene (TNNTI3) locus 19q13.4.

e. Alpha tropomyosin gene (TPM1) locus 15q22.1.

f. Essential light-chain gene (MYL3) locus 3p21.2-p21.3.

g. Alpha-cardiac myosin heavy chain (MYH6) locus 14q11.2-q12.

h. Alpha-cardiac actin (ACTC) locus 15q11-q14.

i. Titin (TTN) locus $2 \mathrm{q} 31$.

j. Regulatory myosine light chain (MYL2) locus 12q23-q.24.3.

k. Cardiac troponin C (TNNC1) locus 3p21.3-p14.3.

2. Z-disc genes:

a. LIM-binding domain 3 (LBD3) locus 10q22.2-q23.3.

b. Muscle LIM protein (CSRP3) locus 11p15.1.

c. Telethonin (TCAP) locus 17q12-q21.1. d. Vinculin (VCL) locus 10q22.1-q23.

e. Alpha-actinin 2 (ACTN2) locus 1q42-q43.

f. Myozenin 2 (MYOZ2) locus 4q26-q27.

3. Calcium-handling genes:

a. Junctophilin-2 (JPH2) locus 20q12.

b. Phospholamban (PLN) locus 6q21.1.

Phenocopies/syndromes with only cardiac manifestation possible. a. AMP-activated protein kinase (PRKAG2) locus 7q35-q36.36 $\rightarrow$ Wolff-Parkinson-White syndrome, with or without HCM CMH.

b. Mitochondrial oxidative phosphorylation (OXPHOS) mtDNA.

c. Alpha-galactosidase A (GLA) locus Xq22 $\rightarrow$ Fabry's disease.

d. Lysosome-associated membrane protein 2 (LAMP2) locus

Xq24 $\rightarrow$ Danon's disease/Wolff-Parkinson-White syndrome.

1.4 OMIM\# of the gene(s)

1. Sarcomeric protein genes:

*600958 Cardiac myosin-binding protein-C gene (MYBPC-3) locus $11 \mathrm{p} 11.2$.

${ }^{+} 160760$ Cardiac beta-myosin heavy chain gene (MYH7) locus 14q11.2-q12.

*191045 Troponin T gene (TNNT2) locus 1q32.

${ }^{+} 191044$ Troponin I gene (TNNTI3) locus 19q13.4.

${ }^{*} 191010$ Alpha tropomyosin gene (TPM1) locus 15q22.1.

${ }^{*} 160790$ Essential light-chain gene (MYL3) locus 3p21.2-p21.3.

${ }^{+} 160710$ Alpha-cardiac myosin heavy chain (MYH6) locus

$14 \mathrm{q} 11.2-\mathrm{q} 12$.

${ }^{+} 102540$ Alpha-cardiac actin (ACTC) locus 15q11-q14.

${ }^{+} 188840$ Titin $(T T N)$ locus 2 q31.

* 160781 Regulatory myosine light chain (MYL2) locus 12q23-q.24.3.

*191040 Cardiac troponin C (TNNC1) locus 3p21.3-p14.3.

2. Z-disc genes:

*605906 LIM domain-binding 3 (LDB3) locus 10q22.2-q23.3.

*600824 Muscle LIM protein (CSRP3) locus 11p15.1.

*604488 Telethonin (TCAP) locus 17q12-q21.1.

*193065 Vinculin (VCL) locus 10q22.1-q23.

* 102573 Alpha-actinin 2 (ACTN2) locus 1q42-q43.

*605602 Myozenin 2 (MYOZ2) locus 4q26-q27.

3. Calcium-handling genes:

*605267 Junctophilin-2 (JPH2) locus 20q12.

*172405 Phospholamban (PLN) locus 6q21.1.

${ }^{1}$ Heart Failure Research Centre, Department of Cardiology, Academic Medical Centre, Amsterdam, The Netherlands; ${ }^{2}$ Department of Clinical Genetics, Academic Medical Centre, Amsterdam, The Netherlands; Inherited Cardiac Diseases Unit, The Heart Hospital (UCL Hospitals NHS trust), University College London, London, UK

${ }^{*}$ Correspondence: Dr YM Pinto, Heart Failure Research Centre, Department of Cardiology and Experimental Cardiology, Academic Medical Centre, Meibergdreef 9,1105 AZ, Amsterdam, The Netherlands.

Tel: +31 205664 688; Fax: +31 206976 177; E-mail: y.pinto@amc.uva.nl 
Phenocopies/syndromes with only cardiac manifestation possible.

${ }^{*} 602743$ AMP-activated protein kinase (PRKAG2) locus 7q35q36.36 $\rightarrow$ Wolff-Parkinson-White syndrome, with our without HCM CMH.

Mitochondrial oxidative phosphorylation (OXPHOS) mtDNA.

*300644 Alpha-galactosidase A (GLA) locus Xq22 $\rightarrow$ Fabry's disease.

*309060 Lysosome-associated membrane protein 2 (LAMP2) locus Xq24 $\rightarrow$ Danon's disease/Wolff-Parkinson-syndrome.

\subsection{Mutational spectrum}

Many genes are involved in HCM. For practically all genes, all types of mutations have been reported (missense, frameshift, nonsense, splice site, and small deletions and insertions).

\subsection{Analytical methods}

Direct sequencing and DHPLC (only for MYBPC3 until the end of 2010). MLPA is also being used.

\subsection{Analytical validation}

Sequencing of both strands. When a mutation is identified, one should validate the mutation in an independent experiment by direct sequencing using a freshly prepared DNA dilution.

\subsection{Estimated frequency of the disease}

(incidence at birth ('birth prevalence') or population prevalence) Estimated that one in 500 individuals is phenotypically affected in the general population worldwide. ${ }^{1-5}$

\subsection{If applicable, prevalence in the ethnic group of investigated person}

There seems to be no clear differences in the HCM prevalence between different ethnicities.

\subsection{Diagnostic setting}

\begin{tabular}{lll}
\hline & Yes. & No. \\
A. (Differential) diagnostics & $\bigotimes$ & $\square$ \\
B. Predictive testing & $\bigotimes$ & $\square$ \\
C. Risk assessment in relatives & $\bigotimes$ & $\square$ \\
D. Prenatal & $\bigotimes$ & $\square$ \\
\hline
\end{tabular}

\section{Comment:}

The two most frequently mutated genes are MYBPC3 and MYH7. ${ }^{6}$ Most of the HCM CMH patients are heterozygous for a mutation, but in $3-5 \%$ of the cases, patients carry two mutations in the same gene (compound heterozygous or homozygous), or in different genes (digenic). In general, this is associated with a more severe phenotype with younger age of onset and more adverse events, suggesting a genedosage effect. ${ }^{6-10}$

\section{TEST CHARACTERISTICS}

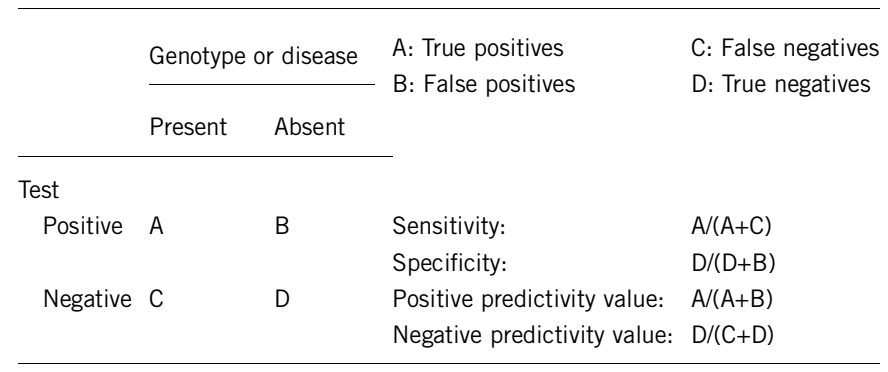

\subsection{Analytical sensitivity}

(proportion of positive tests if the genotype is present)

Almost $100 \%$. Preferential amplification of one allele can happen when one of the primers is located on a SNP. We try to minimise this by checking the primers in SNP databases every other year.

\subsection{Analytical specificity}

(proportion of negative tests if the genotype is not present) Almost 100\%.

\subsection{Clinical sensitivity} (proportion of positive tests if the disease is present)

The clinical sensitivity can be dependent on variable factors such as age or family history. In such cases, a general statement should be given, even if a quantification can only be made case by case.

Many genes involved with mutation frequencies ranging between $30 \%$ and rare (local data and literature). On average, the mutation detection rate for the most obvious set of five candidates is about $56 \%$ (MYBPC3:20-30\%; MYH7:20-30\%; TNNT2:3-5\%; TNNI3:3-5\%; TPM1:1-3\%). ${ }^{11,12,13}$

\subsection{Clinical specificity}

(proportion of negative tests if the disease is not present)

The clinical specificity can be dependent on variable factors such as age or family history. In such cases, a general statement should be given, even if a quantification can only be made case by case.

Probably $100 \%$, but no data available for this measure. Might be a little bit less because of the reduced penetrance of HCM.

\subsection{Positive clinical predictive value}

(life-time risk to develop the disease if the test is positive). Almost $100 \%$ in advanced age. HCM has an age-related pentrance. This penetrance is depending on the individual mutation and/or the gene that is affected and is, in general, steadily increasing until advanced age; see ref. 13 for a review.

\subsection{Negative clinical predictive value} (probability not to develop the disease if the test is negative). Assume an increased risk based on family history for a nonaffected person. Allelic and locus heterogeneity may need to be considered.

Index case in that family had been tested:

If a pathogenic mutation is found in the index and this mutation was not found in another family member, then this member has a $<5 \%$ change of carrying another HCM-causing mutation.

Index case in that family had not been tested:

If the patient has a clinical diagnosis of HCM he/she has a $>90 \%$ chance of having a genetic mutation. Therefore, the chance that another family member will develop HCM once in his/her life will be close to $50 \%$.

\section{CLINICAL UTILITY}

3.1 (Differential) diagnosis: the tested person is clinically affected (To be answered if in 1.10, 'A' was marked) 


\subsubsection{Can a diagnosis be made other than through a genetic test?}

\begin{tabular}{|c|c|c|}
\hline No. & $\square$ (continue with 3.1 .4 ) & \\
\hline \multirow[t]{7}{*}{ Yes. } & $凶$ & \\
\hline & Clinically & $\square$ \\
\hline & Imaging & 凶 \\
\hline & Endoscopy & $\square$ \\
\hline & Biochemistry & $\square$ \\
\hline & Electrophysiology & $\square$ \\
\hline & Other (please describe) & \\
\hline
\end{tabular}

Comment:

The diagnosis of $\mathrm{HCM} \mathrm{CMH}$ is a clinical one, made on the basis of an increased ventricular wall thickness $(15 \mathrm{~mm}$ or more, or $13 \mathrm{~mm}$ or more in relatives of an HCM patient) in the absence of abnormal-loading conditions (arterial hypertension, valve disease), sufficient to cause the observed abnormality. ${ }^{14}$ Wall thickness is assessed using echocardiography or cardiac magnetic resonance imaging. Owing to age-dependant penetrance, a negative clinical test does not exclude the possibility of developing $\mathrm{HCM} \mathrm{CMH}$ at a later age.

\subsubsection{Describe the burden of alternative diagnostic methods to the} patient.

Echocardiography and magnetic resonance imaging are non-invasive procedures with negligible risks and little inconvenience to the patient.

\subsubsection{How is the cost effectiveness of alternative diagnostic methods} to be judged?

When a disease-causing mutation is identified in the index patient, genetic testing can be offered to apparently healthy relatives within the family to determine whether they carry the same mutation and are at risk of developing the disease in the future. If the relative carries the mutation, then regular medical follow-up is required to detect early cardiac disease, and thereby improve their management. Specific advice to gene carriers might include restriction of physical activity (especially competitive sport), avoidance of specific careers, and reproductive counselling.

If a relative does not carry the mutation then they can be reassured and cardiological follow-up discontinued (provided that severity of the phenotype in any family member does not suggest multiple genetic events). Recent evidence indicates that this strategy is highly cost-effective. ${ }^{15}$ An important feature highlighted by this study is the potential for molecular genetic testing to discharge those without a disease-causing mutation who may have had an ambiguous, or even false positive clinical tests requiring ongoing clinical surveillance. It is estimated that by introducing genetic testing, $13 \%$ of patients will be discharged from routine follow-up care.

3.1.4 Will disease management be influenced by the result of a genetic test?

\begin{tabular}{ll}
\hline No. & $\bigotimes$ \\
Yes. & $\square$ \\
& Therapy (please describe) \\
& Prognosis (please describe) \\
& Management (please describe)
\end{tabular}

3.2 Predictive setting: the tested person is clinically unaffected, but carries an increased risk based on family history

(To be answered if in 1.10, 'B' was marked)

\subsubsection{Will the result of a genetic test influence lifestyle and prevention?}

If the test result is positive, regular cardiological evaluation is advised to detect clinical signs of $\mathrm{HCM} \mathrm{CMH}$ and to estimate the risk of sudden cardiac death. Intense physical activity (eg, burst activities, sprinting), or systematic isometric exercise (eg, heavy lifting) will be discouraged.

If the test result is negative (please describe), relatives without the mutation can be discharged form cardiological follow-up. Carriers of mutations will receive reproductive counselling on the risk of transmission to their own offspring.

3.2.2 Which options in view of lifestyle and prevention do a person at-risk have if no genetic test has been done (please describe)?

Advice given will be the same as with a positive genetic test.

3.3 Genetic risk assessment in family members of a diseased person (To be answered if in 1.10 , ' $\mathrm{C}$ ' was marked)

Predictive genetic testing of relatives is only possible if a pathogenic mutation has been identified in an affected person in the family. Autosomal dominant inheritance implies a 50\% risk of being a carrier of the pathogenic mutation in first-degree relatives of a person with the disease.

3.3.1 Does the result of a genetic test resolve the genetic situation in that family?

This is only possible if the mutation in the affected proband is detected (35-65\%). ${ }^{16,17}$ Otherwise, relatives are advised to undergo regular cardiological evaluation.

\subsubsection{Can a genetic test in the index patient save genetic or other tests} in family members?

Yes, it will be cost effective. A negative test result in the index patient does not mean the disease is not hereditary. On the other hand, a positive genetic test result implies the possibility of predictive genetic testing in relatives. Relatives being non-carrier of the pathogenic mutation can be excluded from repeated cardiological follow-up and this will mean saving the costs of ECG and echocardiography. In case of a negative genetic test in the index patient, all relatives are advised to continue regular cardiological follow-up.

3.3.3 Does a positive genetic test result in the index patient enable a predictive test in a family member?

Yes, when a pathogenic mutation is detected.

\subsection{Prenatal diagnosis}

(To be answered if in 1.10 , 'D' was marked)

Prenatal genetic diagnosis in HCM is rarely performed, ${ }^{10}$ and is not actively offered to parents, in common, with other late-onset genetic diseases. Prenatal diagnostics in HCM is only possible when a pathogenic mutation has been detected in the family.

\subsubsection{Does a positive genetic test result in the index patient enable a prenatal diagnostic?}

Yes, but prenatal diagnostics is not actively offered. 


\section{IF APPLICABLE, FURTHER CONSEQUENCES OF TESTING}

Please assume that the result of a genetic test has no immediate medical consequences. Is there any evidence that a genetic test is nevertheless useful for the patient or his/her relatives? (Please describe)

\section{CONFLICT OF INTEREST}

The authors declare no conflict of interest.

\section{ACKNOWLEDGEMENTS}

This work was supported by the EuroGentest, an EU-FP6 supported NoE, contract number 512148 (EuroGentest unit 3: 'Clinical genetics, community genetics, and public health', Workpackage 3.2).

1 Hada $\mathrm{Y}$, Sakamoto $\mathrm{T}$, Amano $\mathrm{K}$ et al: Prevalence of hypertrophic cardiomyopathy in a population of adult Japanese workers as detected by echocardiographic screening. Am J Cardiol 1987; 59: 183-184.

2 Maron BJ, Peterson EE, Maron MS et al: Prevalence of hypertrophic cardiomyopathy in an outpatient population referred for echocardiographic study. Am J Cardiol 1994; 73: 577-580.

3 Maron BJ, Gardin JM, Flack JM et al: Prevalence of hypertrophic cardiomyopathy in a general population of young adults echocardiographic analysis of 4111 subjects in the CARDIA study. Coronary artery risk development in (young) adults. Circulation 1995; 92: 785-789.

4 Zou Y, Song L, Wang Z et al: Prevalence of idiopathic hypertrophic cardiomyopathy in China: a population-based echocardiographic analysis of 8080 adults. Am J Med 2004; 116: 14-18.
5 Morita H, Larson MG, Barr SC et al: Single-gene mutations and increased left ventricular wall thickness in the community: the Framingham Heart Study. Circulation 2006; 113: 2697-2705.

6 Richard P, Charron P, Carrier L et al: Hypertrophic cardiomyopathy: distribution of disease genes, spectrum of mutations, and implications for a molecular diagnosis strategy. Circulation 2003; 107: 2227-2232.

7 Richard P, Isnard R, Carrier L et al: Double heterozygosity for mutations in the betamyosin heavy chain and in the cardiac myosin binding protein $C$ genes in a family with hypertrophic cardiomyopathy. J Med Genet 1999; 36: 542-545.

8 Ho CY, Lever HM, DeSanctis R et al: Homozygous mutation in cardiac troponin T: implications for hypertrophic cardiomyopathy. Circulation 2000; 102: 1950-1955.

9 Richard P, Charron P, Leclercq C et al: Homozygotes for a R869G mutation in the betamyosin heavy chain gene have a severe form of familial hypertrophic cardiomyopathy. J Mol Cell Cardiol 2000; 32: 1575-1583.

10 Lekanne Deprez RH, Muurling-Vlietman JJ, Hruda J et al: Two cases of severe neonatal hypertrophic cardiomyopathy caused by compound heterozygous mutations in the MYBPC3 gene, 2006. J Med Genet 43: 829-832.

11 Marian AJ: Hypertrophic cardiomyopathy: from genetics to treatment. Eur J Clin Invest 2010; 40: 360-369.

12 van Langen Im, Arens Y, Baars $\mathrm{H}$ et al: Genetic diagnostics and genetic counselling in hypertrophic cardiomyopathy (HCM). Neth Heart J 2010; 18: 144.

13 Keren A, Syrris P, McKenna WJ: Hypertrophic cardiomyopathy: the genetic determinants of clinical disease expression. Nat Clin Pract Cardiovasc Med 2008; 5 : 158-168.

14 Elliott PM, Andersson B, Arbustini E et al: Classification of the cardiomyopathies: a position statement from the European Society Of Cardiology Working Group on myocardial and pericardial diseases. Eur Heart J 2008; 29: 270-276.

15 Wordsworth S, Leal J, Blair E et al: DNA testing for hypertrophic cardiomyopathy: a cost-effectiveness model. Eur Heart J 2010; 31: 926-935.

16 Marian AJ, Roberts R: The molecular genetic basis for hypertrophic cardiomyopathy. J Mol Cell Cardiol 2001; 33: 655-670.

17 Van Driest SL, Ommen SR, Tajik AJ et al: Sarcomeric genotyping in hypertrophic cardiomyopathy. Mayo Clin Proc 2005; 80: 463-469. 